\title{
Correlation of Surrogate Markers of Insulin Resistance with Fasting Insulin in Type 2 Diabetes Mellitus Patients: A Study of Malwa Population in Punjab, India
}

\author{
${ }^{1}$ Department of Biochemistry, Guru Gobind Singh Medical College \\ and Hospital, Faridkot, Punjab, India \\ 2Department of Medicine, Guru Gobind Singh Medical College and \\ Hospital, Faridkot, Punjab, India \\ ${ }^{3}$ Department of Radiodiagnosis, Guru Gobind Singh Medical College \\ and Hospital, Faridkot, Punjab, India
}

Navneet Kaur ${ }^{1} \quad$ Gitanjali $^{1} \quad$ Ravinder Garg ${ }^{2} \quad$ Chaitanya Tapasvi $^{3} \quad$ Sonia Chawla ${ }^{1}$ Navdeep Kaur ${ }^{1}$

Address for correspondence Navneet Kaur, MSc, Department of Biochemistry, Guru Gobind Singh Medical College and Hospital, Faridkot 151203, Punjab, India (e-mail: navmann23@yahoo.com).

J Lab Physicians 2021;13:238-244.

\begin{abstract}
Keywords

- serum insulin

- FIRI

- HOMA index

- insulin resistance

- McAuley index

- QUICKI

- type 2 diabetes mellitus

Context Insulin resistance (IR) and abnormal insulin secretion play a key role for the development of type 2 diabetes mellitus (DM).

Aims We investigated the surrogate markers of IR, i.e., Homeostasis Model Assessment (HOMA), Quantitative Insulin Sensitivity Check Index (QUICKI), McAuley, and Fasting Insulin Resistance Index (FIRI) in type 2 DM patients. Also, fasting insulin (FI) levels were estimated in type 2 diabetics. Further, the correlation of FI with other surrogate markers of IR in type 2 DM was done.

Settings and Design A hundred newly diagnosed patients with type 2 DM from Malwa population, Punjab, were considered for evaluation. Another 100 healthy individuals (age and sex-matched) were examined as controls.

Methods and Material Fasting blood glucose, Fl, and lipid profile were estimated, and IR was calculated using McAuley index (McA), HOMA, QUICKI, and FIRI.

Statistical Analysis Used The statistical analysis was performed on the above-mentioned clinical interpretations. The Cohen's kappa test was used to affirm the agreement.

Results FI levels in patients with type 2 diabetes were significantly higher (20.8 $\pm 9.05 \mu \mathrm{IU} / \mathrm{L})$ than controls $(7.93 \pm 1.01 \mu \mathrm{IU} / \mathrm{L})$. IR by surrogate markers was found significant in the study group. The $76 \%$ patients with type 2 diabetes ended up as resistant to insulin by FI measurement, almost equivalent to McA, 80\%; HOMA, 88\%; FIRI, 88\%; and QUICKI, 90\%. A notable correlation was highlighted between FI and McA manifesting IR $(p<0.01, r=-0.85)$. We calculated the statistical correlation of FI with HOMA, QUICKI, and FIRI indices ( $p<0.01, r=0.93 ; p<0.01 r=-0.92$; and $p<0.01, r=+0.93$, respectively). The agreement visible from Cohen's kappa test also affirms the same ( $k=0.9$ for McA). Conclusion We concluded that all the surrogate markers for IR were specific when compared with $\mathrm{Fl}$, but in terms of sensitivity McA was found to be more sensitive as it includes markers of dyslipidemia, which is the precipitating factor of metabolic derangements so as the IR in type $2 \mathrm{DM}$.
\end{abstract}

published online

July 6,2021
DOI https://doi.org/

10.1055/s-0041-1730884

ISSN 0974-2727 (c) 2021. The Indian Association of Laboratory Physicians.

This is an open access article published by Thieme under the terms of the Creative Commons Attribution-NonDerivative-NonCommercial-License, permitting copying and reproduction so long as the original work is given appropriate credit. Contents may not be used for commercial purposes, or adapted, remixed, transformed or built upon. (https://creativecommons.org/licenses/by-nc-nd/4.0/).

Thieme Medical and Scientific Publishers Pvt. Ltd. A-12, 2nd Floor, Sector 2, Noida-201301 UP, India 


\section{Key Message}

The statistical analysis and Cohen's kappa agreement affirm that the McA is sensitive as well as specific to fasting insulin for the insulin resistance evaluation in the Malwa population of Punjab.

\section{Introduction}

Diabetes mellitus (DM), popular as "diabetes", is a condition where a person has elevated blood glucose levels, as the body cells in such persons do not absorb the glucose. This hyperglycemia leads to vascular and other complications. ${ }^{1}$ Type $2 \mathrm{DM}$ is one of the most prevalent types of diabetes, roughly comprising $90 \%$ of the diabetic population across any country. It is higher in developed than in developing countries. According to a survey conducted in 2018, around 500 million global population was diagnosed as type 2 diabetic. ${ }^{2}$ Type $2 \mathrm{DM}$ is a heterogeneous group of disorders with a complex etiology that develops in response to genetic and environmental influences. Insulin resistance (IR) and abnormal insulin secretion are key to the development of type 2 DM. Despite the controversy concerning the primary defect, most of the existing studies embrace the perspective that IR leads up to insulin secretory defects. ${ }^{3}$

IR is one of the common risk markers for type 2 DM. ${ }^{4}$ Studies revealed that $\beta$-cell destruction had already occurred before the patient gets diagnosed with impaired fasting blood glucose levels. ${ }^{1}$ Therefore, IR assessment is very important in the management of DM. The Minimal Model Approximation of the Metabolism of Glucose (MMAMG) is considered as a gold standard method for measuring IR. Other techniques are the euglycemic insulin clamp method and intravenous glucose tolerance test. But these methods are impractical and difficult when adopted in population-based studies. ${ }^{5,6}$ Several indirect methods have also been proposed for the estimation of IR, out of which Homeostasis Model Assessment (HOMA), ${ }^{7}$ Quantitative Insulin Sensitivity Check Index (QUICKI), ${ }^{8}$ McAuley index (McA), ${ }^{9}$ and Fasting Insulin Resistance Index (FIRI) ${ }^{10}$ are widely adopted. Unfortunately, IR is not calculated clinically, excluding the research settings. An enhanced level of fasting blood glucose is the initial indicator of IR. On the contrary, serum fasting insulin (FI) levels are increased much before this actually occurs. The early detection and control are very important to prevent hyperinsulinemia and its associated complications. ${ }^{11}$

FI has been found as an accurate marker for diagnosing IR in the normoglycemic population. ${ }^{12}$ So, the quantification of FI can be adopted as a generic and viable diagnostic marker in contrast to the other indirect techniques used for the diagnosis of IR. So, this study was initiated to determine the role of IR in type 2 diabetics. Here, FI levels were used as a measure of IR. Moreover, the results were compared among each other with the IR value quantified through indirect methods like, McAuley, HOMA, QUICKI, and FIRI.

\section{Subjects and Methods}

The materials and methods used in the present study are discussed in the subsequent subsections.

\section{Study Population}

Keeping in view of the availability and feasibility of the participants, a nonrandom convenient sampling technique was adopted. So, 100 freshly diagnosed type 2 DM patients from Malwa population of Punjab, India who attended the Department of Medicine at Guru Gobind Singh Medical College and Hospital, Faridkot, Punjab, India were considered in our study. Alongside, 100 healthy individuals (age and sex-matched) were taken as controls.

\section{Selection Criteria}

Informed written consent was obtained from study population including controls. The clinical history of the patients (including age, sex, drug, smoking, alcohol consumption, etc.) was obtained from them. According to ADA criteria, patients having fasting plasma glucose $\geq 126 \mathrm{mg} \%$ ( $7 \mathrm{mmol} / \mathrm{L}$ ) and 2-hour OGTT post-load glucose load $\geq 200 \mathrm{mg} \%$ were included in this study. ${ }^{13}$ Patients suffering from any disease of thyroid, liver, kidney or heart failure, stroke, and those having the previous history of insulin treatment were excluded from the study. Pregnant and lactating women were also excluded.

At baseline, in the morning, we sampled the venous blood for the estimation of the blood glucose, triglycerides, total and HDL cholesterol, and FI after a sufficient 10-hour overnight fasting period. Now, the blood glucose was calculated using the glucose oxidase approach. The serum total cholesterol, HDL cholesterol, and triglycerides have been calculated by using standard enzymatic spectrophotometric approaches. The serum LDL cholesterol has been estimated with the Friedewald equation, ${ }^{14}$ except in the case where the triglycerides were more than $400 \mathrm{mg} / \mathrm{dL}$. Special investigations such as serum FI and IR were calculated using various methods. FI was estimated using Accu-Bind Insulin ELISA Kit, a solid phase enzyme-linked immunosorbent assay, that works on the concept of immobilized sandwich. ${ }^{15}$ The variation coefficient for inter- and intraassay was 6.29 and $7.67 \%$, respectively and the sensitivity of assay was $1.5 \mu \mathrm{IU} / \mathrm{L}$.

\section{Data Analysis}

IR was measured using indirect methods HOMA, ${ }^{7}$ QUICKI, ${ }^{8}$ McA, ${ }^{9}$ and FIRI. ${ }^{10}$ The following equations were used to calculate IR using these methods. ${ }^{7-10}$

$$
\begin{aligned}
& \text { HOMA }=\operatorname{insulin}(\mu \mathrm{IU} / \mathrm{L}) \times \frac{\operatorname{glucose}(\mathrm{mmol} / \mathrm{L})}{22.5} \\
& \text { QUICKI }=\frac{1}{\log \text { insulin }+\log \text { glucose }\left(i n \frac{m g}{d L}\right)}
\end{aligned}
$$




$$
\mathrm{McA}=\exp \left[\begin{array}{l}
2.63-0.28 \ln \\
(\text { insulin })(\mu \mathrm{IU} / \mathrm{L}) \\
-0.31 \ln \left(\begin{array}{l}
\text { triglycerides }) \\
(\mathrm{mmol} / \mathrm{L}
\end{array}\right)
\end{array}\right]
$$

FIRI $=$ fasting glucose $(\mathrm{mmol} / \mathrm{L}) \times \frac{\mathrm{FI}(\mu \mathrm{IU} / \mathrm{L})}{25}$

The aforementioned indices were contrasted with FI to investigate the sensitivity and specificity for the prediction of IR. Thereafter, we perform the statistical analysis using the statistical package for social sciences compatible for Windows version 12.0. Alongside, the descriptive analysis was also performed which comprised the calculation of mean values and standard deviations for the continuous variables. Also, the categorical or unequivocal variables were differentiated using the chi-square test. We consider that the $p$-values less than 0.05 are said to be statistically relevant or significant. Correlation was also measured among surrogate markers of IR. Finally, the agreement was measured using Cohen's kappa test to further support the obtained results. ${ }^{16}$

\section{Results}

The results obtained on the basis of considered parameters or markers are presented in the subsequent subsections.

\section{Determinants of IR among Type 2 Diabetics}

Here, the FI levels were significantly higher in the study group, i.e., type 2 diabetic patients $(20.8 \pm 9.05 \mu \mathrm{IU} / \mathrm{L})$ as compared with control subjects $(7.92 \pm 1.01 \mu \mathrm{IU} / \mathrm{L})$. Here, the patients were considered insulin resistant only if $\mathrm{FI} \geq 12 \mu \mathrm{IU} / \mathrm{L} .^{17}$ IR estimated by HOMA, McA, QUICKI, and FIRI techniques was found to be significant in the type 2 DM group. Here, the mean \pm SD values for McAuley, HOMA, QUICKI, and FIRI approaches were estimated as $5.02 \pm 1.23,8.15 \pm 3.92,0.29 \pm$ 0.02 , and $7.33 \pm 3.53$, respectively. We considered the cut-off threshold for a person to be insulin resistant for the aforementioned measures as, McA $\leq 5.8$, HOMA $\geq 2.6$, QUICKI $\leq$ 0.33 , and FIRI $\geq 2.52$ (considering normal reference values of concerned parameters).$^{17}$ - Table 1 presents the baseline characteristics of type 2 diabetics and control groups.

In our study group, a significant negative correlation was witnessed among FI and McA in expressing the IR $(p<0.01$, $r=-0.85)$. - Fig. 1A depicts the correlation of serum insulin levels with IR by McAuley. Moreover, FI is proved to have a statistically relevant correlation with HOMA, QUICKI, and FIRI measures $(p<0.01, r=0.93 ; p<0.01, r=-0.92 ; p<0.01$, $r=+0.93$, respectively). - Fig. 1B depicts the correlation of serum insulin levels with IR by HOMA and - Fig. 1C presents the correlation of serum insulin levels with IR by QUICKI. Lastly, the correlation of serum insulin levels with IR by FIRI is shown in - Fig. 1D. The FI evaluation in type 2 DM patients estimated IR in $76 \%$ patients, quite alike to other practices (McA, 80\%; HOMA, 88\%; QUICKI, 90\%, and FIRI, 88\%).

\section{Prevalence of IR in Different Age Groups}

IR calculated by FI in this study group was grouped according to age. We found out that the age group of 41 to 50 year-old patients contributed the maximum number of diabetic as well as insulin-resistant patients. On the other hand, patients of more than 60 years of age were the least contributor.

\section{Prevalence of IR}

Sensitivity of IR by FI was calculated comparing it with other surrogate markers of IR. Out of type 2 diabetics detected as insulin resistant by McA, 95\% of them were found insulin resistant by FI method. This means that only $5 \%$ of these patients were not detected as insulin resistant by FI. Similarly, among the type 2 diabetics detected as insulin resistant using HOMA and FIRI indices, almost $86.4 \%$ were detected as

Table 1 Baseline characteristics of type 2 diabetics and control groups

\begin{tabular}{|c|c|c|c|c|}
\hline Characteristics & Type 2 DM group & Control group & $p$-Value & Significance \\
\hline Age (years) & $44.6 \pm 10.97$ & $45.74 \pm 8.2$ & $\geq 0.05$ & $\mathrm{NS}^{\mathrm{a}}$ \\
\hline Total cholesterol (mg/dL) & $227.7 \pm 16.6$ & $162.0 \pm 15.2$ & $\leq 0.05$ & $\mathrm{~S}^{\mathrm{b}}$ \\
\hline Triglycerides (mg/dL) & $186.19 \pm 66.2$ & $87.16 \pm 21.6$ & $\leq 0.001$ & $\mathrm{HS}$ \\
\hline HDL cholesterol (mg/dL) & $40.01 \pm 6.01$ & $44.6 \pm 6.09$ & $\leq 0.05$ & $\mathrm{~S}^{\mathrm{b}}$ \\
\hline LDL cholesterol (mg/dL) & $153.19 \pm 6.5$ & $99.4 \pm 11.7$ & $\leq 0.001$ & $\mathrm{HSC}^{\mathrm{c}}$ \\
\hline Fasting blood glucose (mg/dL) & $155.8 \pm 24.3$ & $73.8 \pm 8.6$ & $\leq 0.05$ & $S^{b}$ \\
\hline Fasting insulin ( $\mu \mid \mathrm{U} / \mathrm{L})$ & $20.8 \pm 9.05$ & $7.92 \pm 1.01$ & $\leq 0.001$ & $\mathrm{HS}^{\mathrm{c}}$ \\
\hline McAuley index & $5.02 \pm 1.23$ & $7.95 \pm 0.93$ & $\leq 0.001$ & $H S^{c}$ \\
\hline HOMA index & $8.15 \pm 3.92$ & $1.42 \pm 0.35$ & $\leq 0.001$ & $\mathrm{HS}^{\mathrm{c}}$ \\
\hline QUICKI index & $0.29 \pm 0.02$ & $1.68 \pm 0.13$ & $\leq 0.001$ & $\mathrm{HSc}$ \\
\hline FIRI & $7.33 \pm 3.53$ & $1.04 \pm 0.36$ & $\leq 0.001$ & $H S^{c}$ \\
\hline
\end{tabular}

Abbreviations: DM, diabetes mellitus; FIRI, Fasting Insulin Resistance Index; HDL, high-density lipoprotein; HOMA, Homeostasis Model Assessment; LDL, low-density lipoprotein; QUICKI, Quantitative Insulin Sensitivity Check Index.

aNS, nonsignificant, i.e., $p$-value was $\geq 0.05$.

bS, significant, i.e., $p$-value was $\leq 0.05$.

CHS, highly significant, i.e., $p$-value was $\leq 0.001$. 

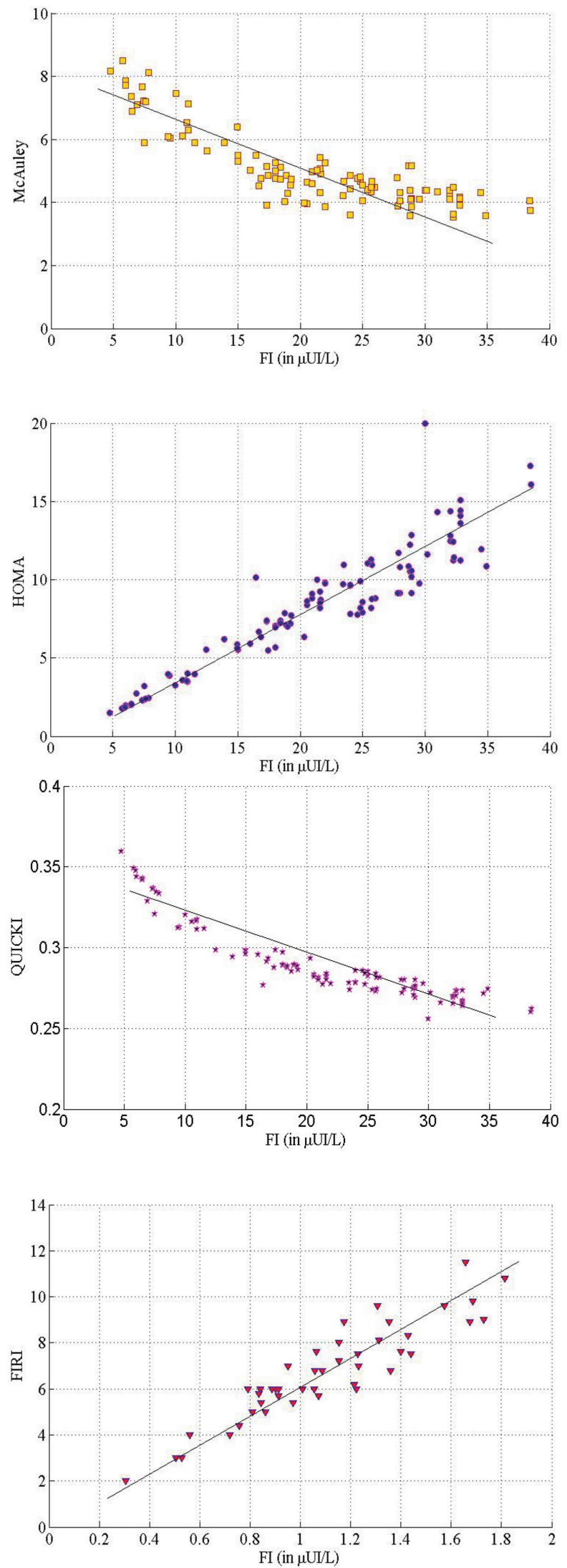

Fig. 1 Correlation plots: (A) Correlation of FI levels with IR by McA, (B) correlation of Fl levels with IR by HOMA, (C) correlation of FI levels with IR by QUICKI, (D) correlation of FI levels with IR by FIRI. FI, fasting insulin; FIRI, Fasting Insulin Resistance Index; HOMA, Homeostasis Model Assessment; IR, insulin resistance; QUICKI, Quantitative Insulin Sensitivity Check Index. insulin resistant when tested using FI method. This indicates that $13.6 \%$ type 2 diabetics detected as insulin resistant using HOMA and FIRI were not insulin resistant when FI test was performed. Also, among all the patients detected by QUICKI, $84.4 \%$ were detected as insulin resistant and further $15.6 \%$ type 2 diabetics were not found as insulin resistant when compared with FI test. - Fig. 2(A-D) shows the pie charts for IR sensitivity as depicted by different surrogate markers.

- Table 2 depict the different metrics (including sensitivity, specificity, accuracy, and Kappa agreement) of FI when compared with surrogate markers. Even the comparison of accuracy for the conducted estimation using four surrogate markers is presented in Fig. 3A. This figure clearly shows that the accuracy for McA is higher in contrast to other surrogate markers. To further support the above results, we conducted Cohen' kappa test to compute the agreement. The agreement values obtained using Cohen's kappa test are also depicted in Fig. 3B. The kappa agreement values also illustrate that there is substantial agreement between FI and McA. In contrast, a moderate agreement is witnessed for HOMA, FIRI, and QUICKI.

\section{Discussion}

In the present study, the relevance of FI as a reliable method for the detection of IR in contrast to the conventional methods such as McAuley, HOMA, QUICKI, and FIRI indices has been assessed. For this reason, recently diagnosed type $2 \mathrm{DM}$ patients were considered to correlate McA, HOMA, QUICKI, and FIRI with FI for IR assessment. Hypertension and hyperinsulinemia occur as a consequence of IR. ${ }^{18}$ Generally, the IR can be estimated using various techniques. But, most of these techniques are not easy to deploy for the clinical practice. As the compensatory hyperinsulinemia is significantly correlated with IR, so it can render more viable way to detect insulin-resistant type 2 diabetics instead of measuring glucose intolerance. ${ }^{19}$

McA is considered as one of the most accurate indirect methods employed to detect IR and more specific as well as sensitive when contrasted with the output depicted by the MMAMG process. Moreover, the specificity as well as the sensitivity of this detection came out to be more for McA. ${ }^{9}$ In the past, it has been discovered that FI test is an accurate marker in normoglycemic population ${ }^{20}$ and the current study demonstrated that the FI test can detect the IR in a diabetic patient in similar significance to McA. The estimation of HOMA-IR shows enhanced sensitivity and specificity for IR specifically for children and adolescents. So, it is demonstrated as a more robust approach than QUICKI. ${ }^{21}$ Another study conducted by Huguette et $\mathrm{a}^{22}$ demonstrated the prevalence of IR by HOMA-IR and QUICKI as 53.9 and 55.7\%, respectively.

Rutter et $\mathrm{al}^{23}$ also demonstrated a relevant correlation for FI and HOMA-IR. Similarly, the results depicted by Conwell et $\mathrm{a}^{24}$ manifested a significant correlation between HOMA-IR and QUICKI, along with sensitivity $(p<0.01)$. They concluded that HOMA-IR, QUICKI, and FI have depicted a strong correlation with sensitivity in obese children and adolescents. 

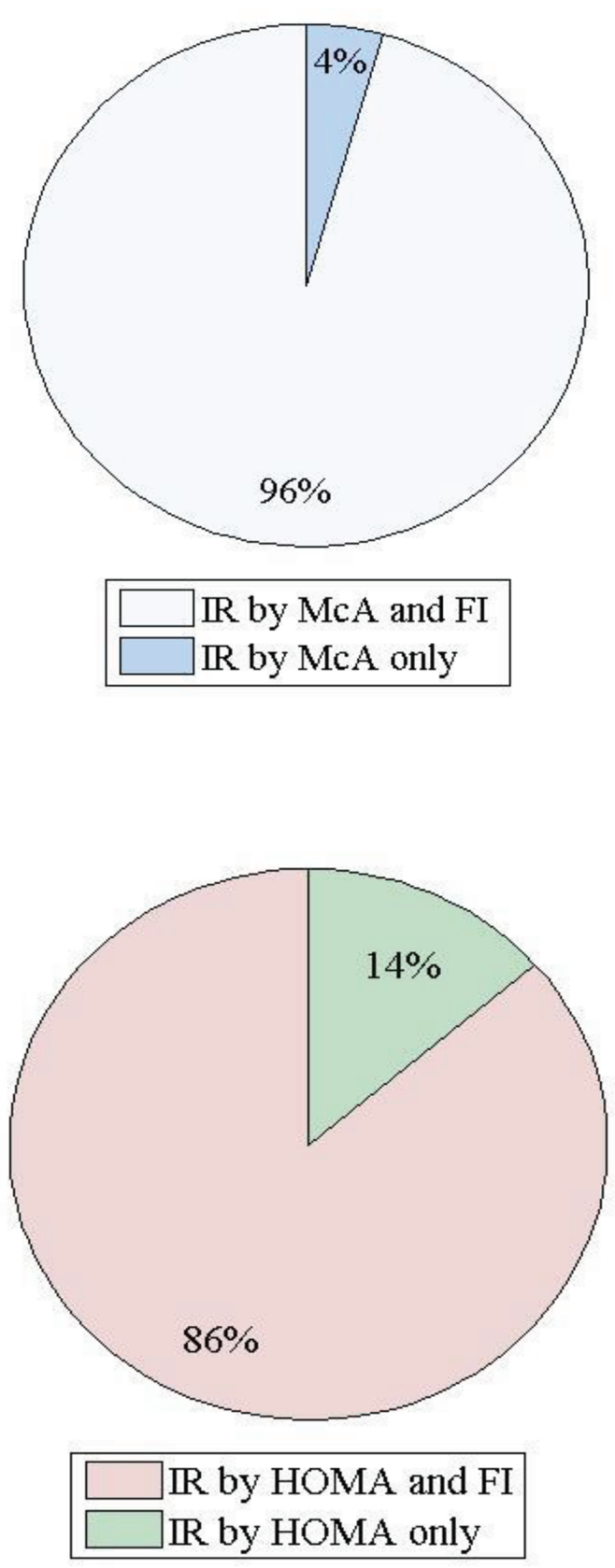

In another study, ${ }^{25}$ all these measures (McA, HOMA, and QUICKI) came out to be highly significant in the diabetic group, and also these parameters correlated well with insulin levels $(p<0.01)$. The FI test showed notable levels of specificity and sensitivity in contrast to McA, QUICKI, and HOMA measures. ${ }^{26}$ In another study, Gates et $\mathrm{al}^{27}$ found FIRI to be significantly correlated with parameters associated with the metabolic syndrome. Moreover, Rudvik, and Månsson ${ }^{28}$ recommended HOMA-IR, QUICKI, and FIRI for IR analysis in clinical studies. All the three parameters had significant correlation with gold standard euglycemic clamp.

In our present study, we have found that $86.36 \%$ patients who proved to be insulin-resistant using HOMA and FIRI indices were also insulin-resistant by FI test. This indicates that $13.6 \%$ of patients detected as insulin-resistant using HOMA and FIRI practices were missed while adopting the

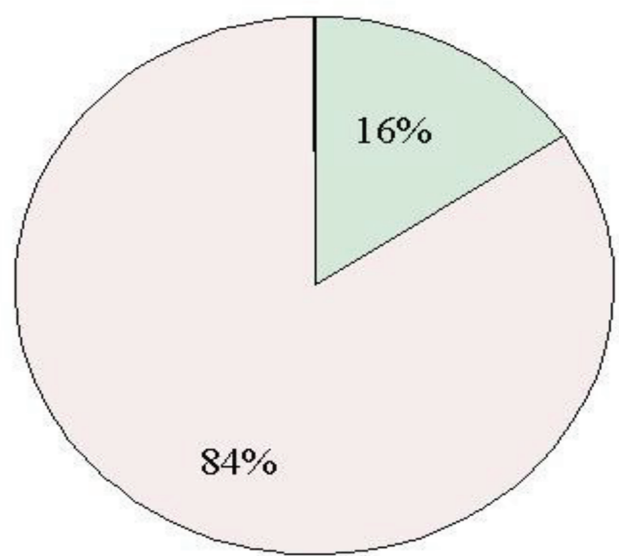

IR by QUICKI and FI IR by QUICKI only

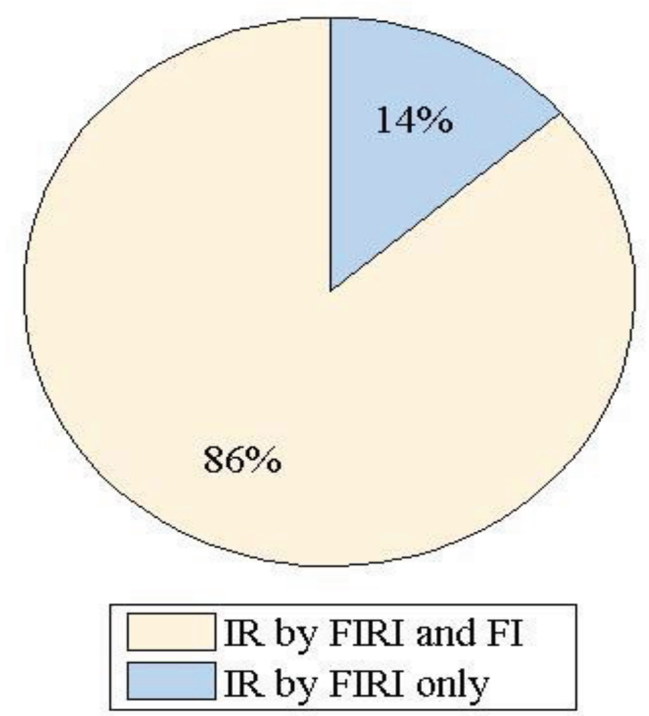

Fig. 2 Sensitivity plots. (A) McA, (B) HOMA, (C) QUICKI, and (D) FIRI. FIRI, Fasting Insulin Resistance Index; HOMA, Homeostasis Model Assessment; McA, McAuley index; QUICKI, Quantitative Insulin Sensitivity Check Index.

FI test. Also, among the people mentioned as insulin resistant by QUICKI, only $84.4 \%$ patients were detected by FI. The primary reason for this indication is supported through the limitations associated with HOMA, QUICKI, and FIRI in the existing studies. HOMA and FIRI are computed using a formula based on fasting glucose and FI therefore it will reflect hepatic insulin sensitivity. ${ }^{29}$ Another study ${ }^{30}$ also supported these outcomes by analyzing the composite insulin resistance which comprises of hepatic as well as peripheral resistance to evaluate the insulin sensitivity in diabetics.

In the present study, the FI test was found to be well correlated with McA, QUICKI, HOMA, and FIRI ( $p<0.005)$. McA was found to be more sensitive marker when compared with FI. The outcomes for the evaluation of IR as depicted through conventional approaches and FI were almost similar. 
Table 2 Sensitivity and specificity of all the methods of insulin resistance

\begin{tabular}{|l|l|l|l|l|l|l|l|l|}
\hline $\begin{array}{l}\text { Methods/ } \\
\text { Parameters }\end{array}$ & TP & FP & TN & FN & Sensitivity (\%) & Specificity (\%) & Accuracy (\%) & kappa \\
\hline McA & 76 & 0 & 20 & 4 & 95 & 100 & 95.56 & 0.90 \\
\hline HOMA & 76 & 0 & 12 & 12 & 86 & 100 & 88 & 0.603 \\
\hline FIR & 76 & 0 & 12 & 12 & 86 & 100 & 88 & 0.603 \\
\hline QUICKI & 76 & 0 & 10 & 14 & 84 & 100 & 86 & 0.521 \\
\hline
\end{tabular}

Abbreviations: FIRI, Fasting Insulin Resistance Index; FN, false negative; FP, false positive; HOMA, Homeostasis Model Assessment; McA, McAuley index; QUICKI, Quantitative Insulin Sensitivity Check Index; TN, true negative; TP, true positive.
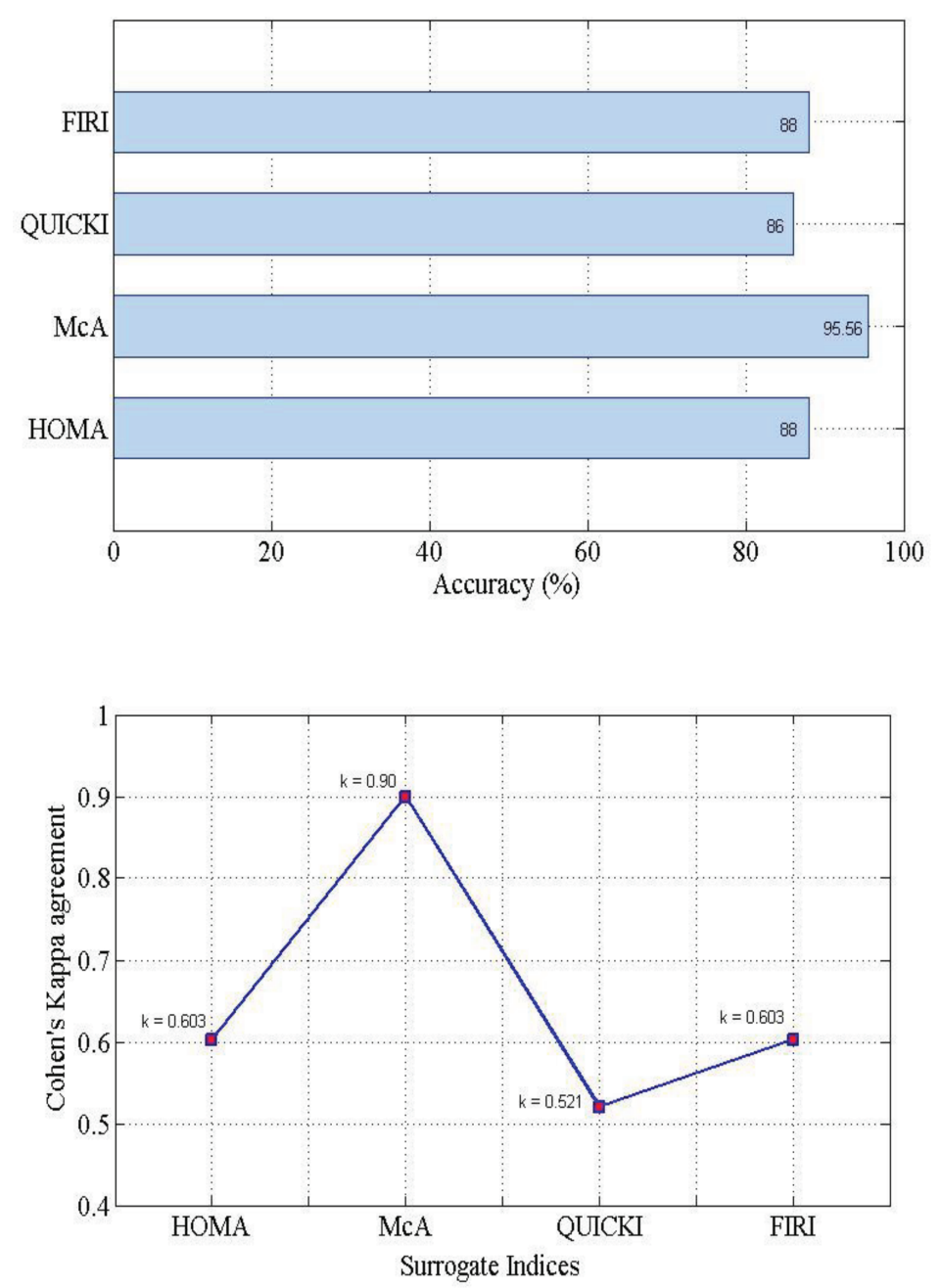

Fig. 3 Comparative plots: (A) Accuracy, and (B) comparison by Chen's Kappa agreement.

Moreover, the viability of McA was evaluated using Cohen's kappa test, which illustrated acceptable and adequate consensus.

\section{Conclusion}

We concluded that all the surrogate markers for IR were specific when compared with $\mathrm{FI}$, but in terms of sensitivity McA was found to be more sensitive as it includes markers of dyslipidemia, which is the precipitating factor of metabolic derangements so as the IR in type $2 \mathrm{DM}$.

\section{Source(s) of Financial Support}

None.

\section{Funding}

No internal or external funding is related to this work.

\section{Ethical Approval}

The approval from the Ethical Committee of the Guru Gobind Singh Medical College and Hospital, Faridkot, Punjab, India, was taken before conducting the present study. 


\section{Conflict of Interest}

None declared.

\section{Acknowledgment}

The authors are thankful to the patients and volunteers involved in the present study to provide their consent. They are also thankful to the clinical staff of Guru Gobind Singh Medical College and Hospital, Faridkot, Punjab, India, for their support during the study.

\section{References}

1 Rother KI. Diabetes treatment-bridging the divide. N Engl J Med 2007;356(15):1499-1501

2 Kaiser AB, Zhang N, Van Der Pluijm W. Global prevalence of type 2 diabetes over the next ten years. 2018;67(suppl 1):1

3 Longo DL, Fauci AS, Kasper DL, et al. Harrison's Principles of Internal Medicine. New York: McGraw-Hill; 2022

4 DeFronzo RA, Ferrannini E. Insulin resistance. A multifaceted syndrome responsible for NIDDM, obesity, hypertension, dyslipidemia, and atherosclerotic cardiovascular disease. Diabetes Care 1991;14(3):173-194

5 Ferrannini E, Mari A. How to measure insulin sensitivity. J Hypertens 1998;16(7):895-906

6 Bergman RN, Finegood DT, Ader M. Assessment of insulin sensitivity in vivo. Endocr Rev 1985;6(1):45-86

7 Matthews DR, Hosker JP, Rudenski AS, Naylor BA, Treacher DF, Turner RC. Homeostasis model assessment: insulin resistance and $\beta$-cell function from fasting plasma glucose and insulin concentrations in man. Diabetologia 1985;28(7):412-419

8 Katz A, Nambi SS, Mather K, et al. Quantitative insulin sensitivity check index: a simple, accurate method for assessing insulin sensitivity in humans. J Clin Endocrinol Metab 2000;85(7):2402-2410

9 McAuley KA, Williams SM, Mann JI, et al. Diagnosing insulin resistance in the general population. Diabetes Care 2001;24(3):460-464

10 Duncan MH, Singh BM, Wise PH, Carter G, Alaghband-Zadeh J. A simple measure of insulin resistance. Lancet 1995;346(8967): 120-121

11 Welch S, Gebhart SS, Bergman RN, Phillips LS. Minimal model analysis of intravenous glucose tolerance test-derived insulin sensitivity in diabetic subjects. J Clin Endocrinol Metab 1990;71(6):1508-1518

12 Thomas GN, Critchley JA, Tomlinson B, Anderson PJ, Lee ZS, Chan JC. Obesity, independent of insulin resistance, is a major determinant of blood pressure in normoglycemic Hong Kong Chinese. Metabolism 2000;49(12):1523-1528

13 American Diabetes Association. Classification and diagnosis of diabetes-2020. Diabetes Care 2020;43(1):14-31

14 Friedewald WT, Levy RI, Fredrickson DS. Estimation of the concentration of low-density lipoprotein cholesterol in plasma, without use of the preparative ultracentrifuge. Clin Chem 1972;18(6):499-502

15 Gerbitz K. Pancreatic b-cell peptides: kinetics and concentration of proinsulin, insulin and c-peptide in plasma and urine, problems with measurement methods, clinical information and literature "u overview. Clin Chem Lab Med 1980;18:313-326
16 Berry KJ, Mielke PW Jr. A generalization of Cohen's kappa agreement measure to interval measurement and multiple raters. Educ Psychol Meas 1988;48:921-933

17 Matsuda M, DeFronzo RA. Insulin sensitivity indices obtained from oral glucose tolerance testing: comparison with the euglycemic insulin clamp. Diabetes Care 1999;22(9):1462-1470

18 Bano KA, Begum M, Hussain R. Fasting blood level of insulin in non-obese and non-diabetic patients with essential hypertension. Pak J Med Res 2004;43:5-7

19 Laakso M. How good a marker is insulin level for insulin resistance? Am J Epidemiol 1993;137(9):959-965

20 Mack R, Skurnick B, Sterling-Jean Y, Pedra-Nobre M, Bigg D. Fasting insulin levels as a measure of insulin resistance in American Blacks. J Med 2003;34(1-6):31-38

21 Simsek E, Karabay M, Aras S, Kocabay K. Investigating for insulin resistance and type 2 diabetes mellitus in obese children. Turk J Endocrinol Metab 2005;1:17-22

22 Huguette YMC, Boris AKG, Raıssa NF, Laure NJ, Julius O. Prevalence of insulin resistance in obese Cameroonian women. J Diab Endocrinol 2010;1:19-26

23 Rutter MK, Wilson PW, Sullivan LM, Fox CS, D’Agostino RB Sr, Meigs JB. Use of alternative thresholds defining insulin resistance to predict incident type 2 diabetes mellitus and cardiovascular disease. Circulation 2008;117(8):1003-1009

24 Conwell LS, Trost SG, Brown WJ, Batch JA. Indexes of insulin resistance and secretion in obese children and adolescents: a validation study. Diabetes Care 2004;27(2):314-319

25 Hettihewa LM, Palangasinghe S, Jayasinghe SS, Gunasekara SW, Weerarathna TP. Comparison of insulin resistance by indirect methods-HOMA, QUICKI, and McAuley- with fasting insulin in patients with type 2 diabetes in Gale, Sri Lanka: a Pilot Study. Outline J Health Allied Sci 2006;5(1) Hettihewa LM, Palangasinghe S, Jayasinghe SS, Gunasekara SW, Weerarathna TP. Comparison of insulin resistance by indirect methods-HOMA, QUICKI, and McAuley- with fasting insulin in patients with type 2 diabetes in Gale, Sri Lanka: a Pilot Study. Outline J Health Allied Sci 2006;5(1):1-8

26 Garg MK, Tandon N, Marwaha RK, Singh Y. Evaluation of surrogate markers for insulin resistance for defining metabolic syndrome in urban Indian adolescents. Indian pediatrics 2014;51(4):279-284

27 Gates JR, Parpia B, Campbell TC, Chen J. Evaluation of the FIRI (Fasting Insulin Resistance Index) and selected plasma parameters associated with insulin resistance as predictors of cardiovascular mortality in rural Chinese women. Asia Pac J Clin Nutr 1997;6(3):200-202

28 Rudvik A, Månsson M. Evaluation of surrogate measures of insulin sensitivity-correlation with gold standard is not enough. BMC Med Res Methodol 2018;18(1):64

29 Bonora E, Targher G, Alberiche M, et al. Homeostasis model assessment closely mirrors the glucose clamp technique in the assessment of insulin sensitivity: studies in subjects with various degrees of glucose tolerance and insulin sensitivity. Diabetes Care 2000;23(1):57-63

30 Miyazaki Y, Mahankali A, Wajcberg E, Bajaj M, Mandarino LJ, DeFronzo RA. Effect of pioglitazone on circulating adipocytokine levels and insulin sensitivity in type 2 diabetic patients. The Journal of Clinical Endocrinology\& Metabolism 2004; 89(9):4312-4319 